\section{Physicist whose work led to the discovery of the cosmic microwave background}

In the exuberant world of present-day gravity physics, it is hard to picture research four decades ago when you could count the active people on your fingers. Bob Dicke, who died on 4 March, was a leader in this change, most famously in the discovery of thermal radiation from the Big Bang, which was detected by a Dicke radiometer and interpreted as part of his programme of improving the empirical basis for gravity physics.

Dicke's radiometer was a product of wartime work on radar at the MIT Radiation Laboratory. Its Dicke switch, which vastly improves sensitivity by comparing the signal with a stable reference, is a standard tool for radioastronomy. The radiometer showed that collisions broaden the absorption lines of atmospheric water vapour, explaining why the wartime drive to shorter wavelength for better radar resolution failed at one centimetre. It detected thermal radiation from the Moon, and showed that space could not be warmer than $20 \mathrm{~K}$ above absolute zero. Two decades later, Dicke radiometers showed that the temperature of the present-day Universe is actually $2.7 \mathrm{~K}$.

After the war, Dicke continued his work on quantum optics at Princeton University. He showed that inert atoms added to a gas cell suppress first-order Doppler shifts by shortening the mean free path of radiating atoms, even though they have no effect on the thermal velocities. The physics is the same as in the Mössbauer effect: as in a crystal lattice, the confinement allows the atom only discrete values of kinetic energy, and unless the recoil is strong enough to force a quantum jump, the emitted photon gets all the decay energy.

In the mid-1950s Dicke turned to gravity physics. At the time, Einstein's general relativity was the accepted theory of gravity, but Dicke recognized that the experimental basis for this theory was weak and could be considerably improved by applying the great advances in technology of measurements that grew out of war research. Dicke's improvement of the classic Eötvös experiment showed that the gravitational accelerations of different test masses agree to three parts in $10^{11}$. Another experiment, with his student Lloyd Kreuzer, used a body floating in a

\section{IMAGE UNAVAILABLE FOR COPYRIGHT REASONS}

fluid of a different composition to test the equivalence of passive and active gravitational mass (respectively the response to and the source of gravity). At neutral buoyancy, the passive mass densities are the same in the body and the fluid. The lack of a detectable change in the active mass distribution when the body is moved in the fluid shows that the two measures of mass are very nearly the same.

Dicke was fascinated by the idea that the strength of gravity might be evolving, and considered many tests for the effect. For example, a stronger gravitational interaction would cause faster nuclear burning in stars, possibly accounting for the fact that some stars appeared older than the expansion time since the Big Bang. With Carl Brans, a student of John Wheeler, Dicke proposed a generalized theory in which the strength of the gravitational interaction decreases as the Universe expands. Variants of the Brans-Dicke theory figure in models for the very early Universe, when it was tens of orders of magnitude smaller than it is now, but no longer for gravity physics at the present epoch. Current results from tests that Dicke and a few others pioneered in the 1950s, including the deflection and frequency shift of radiation near mass concentrations and the motions of planets and pulsars, are consistent with general relativity rather than the Brans-Dicke generalization.

Still deeper probes of general relativity are under way, using the condition that the distribution of the thermal radiation from the Big Bang should be consistent with that of the mass fluctuations traced by the galaxies. In the mid-1960s, Dicke led in the discovery of the key to this test, the thermal radiation. At the time, he was at most only vaguely aware of work suggesting a hot Big Bang. Richard Tolman had shown that if the Universe expanded from a hot dense state it would be filled with thermal radiation that cools with the expansion but keeps its characteristic thermal spectrum. George Gamow added to the picture by showing that thermonuclear reactions in a hot young Universe could produce a reasonable helium abundance. Ralph Alpher and Robert Herman refined Gamow's estimate and extrapolated it to predict the present temperature, $5 \mathrm{~K}$, not far from the actual value of $2.7 \mathrm{~K}$. A measurement did not occur to Gamow's group. It did to Dicke, who was following another idea.

In a high-density Universe, gravity eventually stops the expansion. The Universe then collapses, and if the collapse ends in a bounce and another expansion, we have an oscillating Universe. The bounce might thermalize starlight from the last cycle. Dicke persuaded Peter Roll and one of us (D.T.W.) to build a radiometer, improved over the original by a liquid-helium reference for absolute calibration, to look for this fossil. D.T.W. gave the other of us leave to present a colloquium on the search and its significance (on the grounds that the experiment would be done before any competition could get started). Word travelled from the symposium through yet another of Dicke's former students, Ken Turner, eventually to Arno Penzias and Robert Wilson at Bell Labs, who had been seeking the cause of unexpected noise in a Dicke radiometer aimed at the sky. News of the Roll-Wilkinson experiment led them to think the noise might be extraterrestrial. The Princeton measurement, at half the wavelength of the Bell Labs result, soon gave evidence that the spectrum is thermal, as expected if this is the fossil of the Big Bang (whether or not it is thermalized starlight). And out of this grew a small industry, studying our Universe as it is now and used to be.

Bob Dicke was an enthusiastic pianist, but he loved science even more. His frequent request was, "tell me something new". In the early days he held research seminars on Friday evenings; we complained but attended because the physics was too fascinating to miss. He probably knew that we called ourselves Dicke-birds - it fitted his quiet good humour, which kept us from taking ourselves too seriously while always remembering that we had better take the physics very seriously.

\section{P. J. E. Peebles and D. T. Wilkinson}

P. J. E. Peebles and D. T. Wilkinson are in the Department of Physics, Princeton University, Princeton, New Jersey 80544, USA. 